Copyright(C)2005 IEEE. Reprinted from:

C. B. Ribeiro, E. Ollila, and V. Koivunen, "Propagation parameter estimation in MIMO systems using mixture of angular distributions model," in Proc. IEEE Int. Conf. on Acoustics, Speech and Signal Processing, ICASSP 2005, Mar. 2005, vol. 4, pp. 885-888.

This material is posted here with permission of the IEEE. Such permission of the IEEE does not in any way imply IEEE endorsement of any of the Helsinki University of Technology's products or services. Internal or personal use of this material is permitted. However, permission to reprint/republish this material for advertising or promotional purposes or for creating new collective works for resale or redistribution must be obtained from the IEEE by writing to pubs-permissions@ieee.org.

By choosing to view this material, you agree to all provisions of the copyright laws protecting it. 


\title{
PROPAGATION PARAMETER ESTIMATION IN MIMO SYSTEMS USING MIXTURE OF ANGULAR DISTRIBUTIONS MODEL
}

\author{
Cássio B. Ribeiro*, Esa Ollila and Visa Koivunen \\ Signal Processing Laboratory, SMARAD CoE \\ Helsinki University of Technology \\ PO box 3000, FIN-02015 HUT, Finland
}

\begin{abstract}
For the development of future wireless systems, it is crucial to create accurate channel models. Channel sounding using antenna arrays and consequently propagation parameter estimation are key tasks in creating such models. In this paper we present an estimator for the angular distribution of the diffuse scattering component that is observed in channel sounding measurements. The angular distribution is modeled as a mixture of Von Mises distributions, which correspond to scatterer clusters. The parameters of the individual distributions as well as the mixture proportions are estimated. The large sample performance of the estimator is studied by deriving the Cramér-Rao lower bound and comparing the variance of the estimates to it. The simulations show that the the proposed estimator has asymptotically optimal performance since it attains the Cramér-Rao lower bound for relatively small sample sizes.
\end{abstract}

\section{INTRODUCTION}

In radio propagation it is usual to classify the signals that reach the receiver as been originated by specular reflections or scattering. The specular components usually carry most of the power, and are modeled by a relatively large number of deterministic signals with unknown parameters [1]. Scattering is frequently regarded as noise and neglected. However, even though each scattered wave arrives with low power, the overall sum of scattering components can be significant, and even dominant, especially in non line-ofsight (NLOS) situations. This behavior has been observed in measurement campaigns such as [2].

Deterministic techniques for propagation parameter estimation commonly employ models with large number of discrete waves. This approach leads to maximization of highly non-linear likelihood functions with many local optima, which causes convergence problems [3]. The computational complexity and variance of estimates are increased, since a large number of parameters has to be estimated.

Typically isotropic scattering models are employed in channel models. The channel model considered in this work is also suitable for non-isotropic scattering model and nonuniform distribution of angles of arrival. The model stems from the MIMO channel correlation model presented in [4]. The MIMO channel matrix may be described analytically as a function of the parameters of the underlying random processes.

* This work also supported by Conselho Nacional de Desenvolvimento Científico e Tecnológico - CNPq, Brazil and Academy of Finland.
In this paper we derive estimators for the channel propagation parameters employing angular distribution models, in particular the Von Mises distributions (see, [5]). Also other angular distribution models could be used. The parameters of the diffuse scattering component in MIMO systems are of interest. The proposed method extends the method presented in $[6,7]$ to demanding scattering environments where there may be multiple clusters of scatterers present. Typical example of such environment is the bad urban scenario in mobile wireless communications. A mixture of Von Mises distributions is employed in order to estimate the propagation parameters in scattering environment with multiple clusters of scatterers with high fidelity.

The large sample properties of the proposed estimation method are established. We derive the Cramér-Rao Lower Bound (CRLB) for the mixture model considered in this article. This is an extension of the result in [7] to multiple clusters. The variance of the obtained estimates is compared to the CRLB. The proposed estimator has desirable optimality properties since it attains the CRLB with relatively small sample sizes. Moreover, it is numerically more stable than the methods using deterministic models with large number of unknown parameters, e.g. [3]. The proposed method may be used to develop and validate realistic channel models for multiantenna communications as well as estimate parameters in other sensor array applications.

This paper is organized as follows: in Section 2 we describe the signal model used in this article. In Section 3 the technique for parameter estimation is described. In Section 4 the Cramér-Rao lower bound is established. Finally, in Section 5 we present some simulation results and compare the large sample performance of the estimation technique to the CRLB.

\section{SIGNAL MODEL}

The transmitter is assumed to be elevated and therefore not obstructed by local scatterers, while the receiver is surrounded by a large number of local scatterers. No line-of-sight is assumed between the transmitter and the receiver. We consider that the waves are planar (far-field) and the "one-ring" scatterer model is used, as in [4] to study the effect of fading correlation on the capacity of MIMO fading channels.

Let us define the output of the each antenna at the receiver by the $N \times 1$ vector $\mathbf{y}(k)$, the transmitted sequences by the $M \times 1$ vector $\mathbf{u}(k)$, the $N \times M$ MIMO channel matrix $\mathbf{H}(k)$, and the $N \times 1$ vector $\mathbf{n}(k)$ with random noise. The signals defined above are discrete-time versions of continuous-time signals sampled at time instants $k T_{s}$, where $T_{s}$ is the sampling period. Using these 
definitions we model the received signal as

$$
\mathbf{y}(k)=\mathbf{H}(k) \mathbf{u}(k)+\mathbf{n}(k) .
$$

In this model it is assumed that the signal bandwidth is sufficiently narrow so that the channel can be considered frequency non-selective.

The channel sounding technique assumed in this work is based on time division multiplexing of each transmitter and receiver antenna, like in PropSound channel sounder $[1,8]$. This particular structure makes it is possible to separate the contribution from each transmitter and receiver antenna.

The channel matrix thus defined represents the combination of all waves that impinge the receiver array after being reflected by the surrounding scatterers. Deterministic estimation techniques such as SAGE $[1,8]$ represents the received signal as a combination of several discrete waves. Consequently parameters from a large number of waves must be estimated. Hence, the algorithms often have convergence problems due to local minima in likelihood function.

In [4] the authors present a MIMO channel model whose correlation matrix can be described analytically, and thus can be used to derive estimators for the channel parameters. In this model the signals are considered as realizations of an underlying random process and not as deterministic signals. The authors derived a closed form solution for the cross-correlation between any two sub-channels assuming that the incident angles follow a Von Mises distribution [5], but other distributions can also be used.

\section{PROPAGATION PARAMETER ESTIMATION}

The following assumptions will be employed throughout this article:

(a) switched multiple element transmit antenna;

(b) the received signal $\mathbf{y}(k)$ is a zero-mean circular complex Gaussian process;

(c) channel is stationary during one measurement cycle;

(d) the additive noise $\mathbf{n}(k)$ is a zero-mean circular complex Gaussian process independent of $\mathbf{H}(k)$.

(e) the variances, $E\left[\left|h_{l}\right|^{2}\right]$, for each MIMO subchannel $h_{l}$ are equal and assumed to be known. We denote this variance by $\Omega$ and call it the path-loss.

From assumption (a), if one transmit antenna is considered at the time, the channel matrix $\mathbf{H}(k)$ in (1) is a $N \times 1$ vector denoted by lowercase $\mathbf{h}(k)$. From assumption (b), the pdf of the received signal $\mathbf{y}(k)$ is completely characterized by its covariance matrix

$$
\mathbf{C}_{y}=E\left[\mathbf{y}(k) \mathbf{y}^{H}(k)\right] .
$$

From (1) we can write $\mathbf{C}_{y}$ as

$$
\mathbf{C}_{y}=E\left[\mathbf{h h}^{H}\right] P_{u}+\mathbf{C}_{n},
$$

where $\mathbf{C}_{n}$ is the noise covariance matrix, and $P_{u}=|u(k)|^{2}$.

Using the channel model in [4] we can write $E\left[\mathbf{h} \mathbf{h}^{H}\right]$ as a function of the channel parameters. It is assumed that the receiver is surrounded by a large number of local scatterers, and that the waves reflected by different scatterers arrive at the array with the same power. This situation can be represented as a ring of scatterers around the receiver, as depicted in Figure 1 for any 2 antennas at the transmitter and receiver.

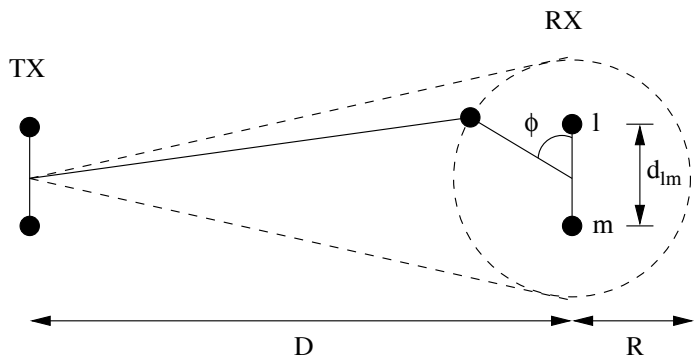

Fig. 1. Illustration of the geometrical configuration of a $2 \times 2$ channel with local scatterers at the receiver, where $D$ is the distance between the transmitter and receiver arrays, $R$ is the radius of the ring of scatterers, and $d_{l m}$ is the distance between elements $l$ and $m$ in the receive array.

Assuming that the angle spread at the transmitter is small and $D \gg R \gg d_{l m}$, it is possible to show that the cross-correlation between any two sub-channels $l$ and $m$ is given by [4]

$$
\rho_{l m}=\frac{1}{\Omega} E\left[h_{l} h_{m}^{*}\right]=\int_{-\pi}^{\pi} \exp \left(b_{l m} \cos (\phi)\right) f(\phi) d \phi,
$$

where $f(\phi)$ is any angular PDF of $\phi, b_{l m}=j 2 \pi d_{l m} / \lambda, d_{l m}$ is the distance between elements $l$ and $m$ in the receive array, $\lambda$ is the transmitted signal wavelength, and $h_{l}$ is the $l$-th element of $\mathbf{h}$.

\subsection{Multiple Clusters}

In channel measurements it is often found that the signal is received as coming from a number of different clusters, specially in bad urban scenarios. This is equivalent to a situation where the angular pdf $f(\phi)$ in equation (3) is a mixture of distributions,

$$
f(\phi)=\sum_{p=1}^{P} \epsilon_{p} f_{p}(\phi),
$$

where $P$ is the number of clusters, $\sum_{p=1}^{P} \epsilon_{p}=1, \epsilon_{p}$ are unknown mixture proportions, and $f_{p}(\phi)$ is any valid angular pdf. A suitable angular pdf is the Von Mises [5], defined as

$$
f(\phi)=\frac{1}{2 \pi I_{0}(\kappa)} \exp (\kappa \cos (\phi-\mu)),
$$

where $\mu$ is the symmetry center and $\kappa$ can be chosen between 0 (isotropic scattering) and $\infty$ (extremely concentrated).

Using (4) and (5), the cross correlation in (3) may be written analytically as $[4,6]$

$$
\rho_{l m}=\sum_{p=1}^{P} \epsilon_{p} \frac{I_{0}\left(\left\{\kappa_{p}^{2}+b_{l m}^{2}+2 \kappa_{p} b_{l m} \cos \left(\mu_{p}\right)\right\}^{\frac{1}{2}}\right)}{I_{0}\left(\kappa_{p}\right)} .
$$

The ML estimates of $\theta_{p}=\left\{\kappa_{p}, \mu_{p}, \epsilon_{p}\right\}, p=1, \ldots, P$, are those values that maximize the likelihood function. After removing the constant terms not dependent on the signal, the loglikelihood function can be written as

$$
\mathcal{L}\{\boldsymbol{\theta}\} \propto-\log \left|\mathbf{C}_{y}\right|-\operatorname{tr}\left\{\mathbf{C}_{y}^{-1} \widehat{\mathbf{C}}_{y}\right\}
$$


where $\widehat{\mathbf{C}}_{y}$ is the sample covariance matrix, defined as $\widehat{\mathbf{C}}_{y}=$ $\left(1 / N_{s}\right) \sum_{k=0}^{N_{s}-1} \mathbf{y}(k) \mathbf{y}^{H}(k)$, and $\operatorname{tr}\{\cdot\}$ denotes the trace. The ML estimates are obtained as

$$
\begin{aligned}
\widehat{\boldsymbol{\theta}}= & \operatorname{argmax}_{\boldsymbol{\theta}}\left\{-\log \left|\mathbf{C}_{y}\right|-\operatorname{tr}\left\{\mathbf{C}_{y}^{-1} \widehat{\mathbf{C}}_{y}\right\}\right\}, \\
& \text { s.t. } \sum_{p=1}^{P} \epsilon_{p}=1
\end{aligned}
$$

where $\boldsymbol{\theta}=\left[\begin{array}{lll}\theta_{1} & \cdots & \theta_{P}\end{array}\right]^{T}$. In order to optimize (8), we will use a Sequential Quadratic Programming algorithm [9], as implemented by the fmincon function of Matlab.

\section{CRAMÉR-RAO BOUND}

The Fisher information matrix can be derived for the multiple cluster as an extension of the Fisher information matrix for the single cluster case. The log-likelihood equation is given by

$$
\mathcal{L}\{\boldsymbol{\theta}\}=-N_{s} \log \pi^{N}-N_{s} \log \left|\mathbf{C}_{y}\right|-N_{s} \operatorname{tr}\left\{\mathbf{C}_{y}^{-1} \widehat{\mathbf{C}}_{y}\right\} .
$$

We can then calculate

$$
\begin{aligned}
d \mathcal{L}\{\boldsymbol{\theta}\} & =-N_{s} d \log \left|\mathbf{C}_{y}\right|-N_{s} \operatorname{tr}\left\{d \mathbf{C}_{y}^{-1} \widehat{\mathbf{C}}_{y}\right\} \\
& =-N_{s} \operatorname{tr}\left\{\left(\mathbf{I}-\mathbf{C}_{y} \widehat{\mathbf{C}}_{y}\right) \mathbf{C}_{y}^{-1}\left(d \mathbf{C}_{y}\right\}\right.
\end{aligned}
$$

From (6), the elements of the derivatives of $\mathbf{C}_{y}(\boldsymbol{\theta})$ with respect to $\mu_{p}$ and $\kappa_{p}, p=1, \ldots, P$, are given by

$$
\begin{aligned}
\left\{\mathbf{D}_{\mu_{p}}\right\}_{l, m}= & -\epsilon_{p} \Omega P_{u} I_{0}^{-1}\left(\kappa_{p}\right) I_{1}\left(\beta_{p}\right) \beta_{p}^{-1} \kappa_{p} b_{l m} \sin \mu_{p} \\
\left\{\mathbf{D}_{\kappa_{p}}\right\}_{l, m}= & \epsilon_{p} \Omega P_{u}\left[-I_{0}^{-2}\left(\kappa_{p}\right) I_{1}\left(\kappa_{p}\right) I_{0}\left(\beta_{p}\right)+\right. \\
& \left.+I_{0}^{-1}\left(\kappa_{p}\right) I_{1}\left(\beta_{p}\right) \beta_{p}^{-1}\left(\kappa_{p}+b_{l m} \cos \mu_{p}\right)\right]
\end{aligned}
$$

where $\beta_{p}=\left(\kappa_{p}^{2}+b_{l m}^{2}+2 \kappa_{p} b_{l m} \cos \mu_{p}\right)^{1 / 2}, I_{0}(\cdot)$ and $I_{1}(\cdot)$ are the modified Bessel function of the first kind of order zero and one, respectively. The condition $\sum_{p=1}^{P} \epsilon_{p}=1$ can be rewritten as $\epsilon_{P}=1-\sum_{p=1}^{P-1} \epsilon_{p}$, and then we can calculate the derivatives of $\mathbf{C}_{y}(\boldsymbol{\theta})$ with respect to $\epsilon_{p}, p=1, \ldots, P-1$, as

$$
\left\{\mathbf{D}_{\epsilon_{p}}\right\}_{l, m}=\Omega P_{u}\left[I_{0}^{-1}\left(\kappa_{p}\right) I_{0}\left(\beta_{p}\right)-I_{0}^{-1}\left(\kappa_{P}\right) I_{0}\left(\beta_{P}\right)\right] \text {. }
$$

In order to simplify the notation we will write $\mathbf{D}_{i}=\mathbf{D}_{\boldsymbol{\theta}_{i}}$, $i=1, \ldots, 3 P-1$. Hence, using equations (10)-(13), we can write

$$
\frac{\partial \mathcal{L}\{\boldsymbol{\theta}\}}{\partial \boldsymbol{\theta}_{i}}=-N_{s} \operatorname{tr}\left\{\left(\mathbf{I}_{N}-\mathbf{C}_{y}^{-1} \widehat{\mathbf{C}}_{y}\right) \mathbf{C}_{y}^{-1} \mathbf{D}_{i}\right\}
$$

We obtain the second derivatives by differentiating (14) with respect to $\boldsymbol{\theta}_{j}$ as

$$
\begin{aligned}
\frac{\partial^{2} \mathcal{L}\{\boldsymbol{\theta}\}}{\partial \boldsymbol{\theta}_{i} \partial \boldsymbol{\theta}_{j}}= & -N_{s} \operatorname{tr}\left\{\mathbf{C}_{y}^{-1} \mathbf{D}_{j} \mathbf{C}_{y}^{-1} \widehat{\mathbf{C}}_{y} \mathbf{C}_{y}^{-1} \mathbf{D}_{i}+\right. \\
& \left.+\left(\mathbf{I}_{N}-\mathbf{C}_{y}^{-1} \widehat{\mathbf{C}}_{y}\right)\left[\partial\left(\mathbf{C}_{y}^{-1} \mathbf{D}_{i}\right) / \partial \boldsymbol{\theta}_{j}\right]\right\},
\end{aligned}
$$

We can then write

$$
E\left[\frac{\partial^{2} \mathcal{L}\{\boldsymbol{\theta}\}}{\partial \boldsymbol{\theta}_{i} \partial \boldsymbol{\theta}_{j}}\right]=-N_{s} \operatorname{tr}\left\{\mathbf{C}_{y}^{-1} \mathbf{D}_{j} \mathbf{C}_{y}^{-1} \mathbf{D}_{i}\right\},
$$

where it is used the fact that $E\left[\widehat{\mathbf{C}}_{y}\right]=\mathbf{C}_{y}$. The $\{i, j\}$ element of the Fisher information matrix is then given by

$$
\{\mathbf{I}\}_{i, j}=N_{s} \operatorname{tr}\left\{\mathbf{C}_{y}^{-1} \mathbf{D}_{j} \mathbf{C}_{y}^{-1} \mathbf{D}_{i}\right\}
$$

The Cramer-Rao bounds can then be evaluated for each parameter set as the elements in the diagonal of the inverse Fisher information matrix.

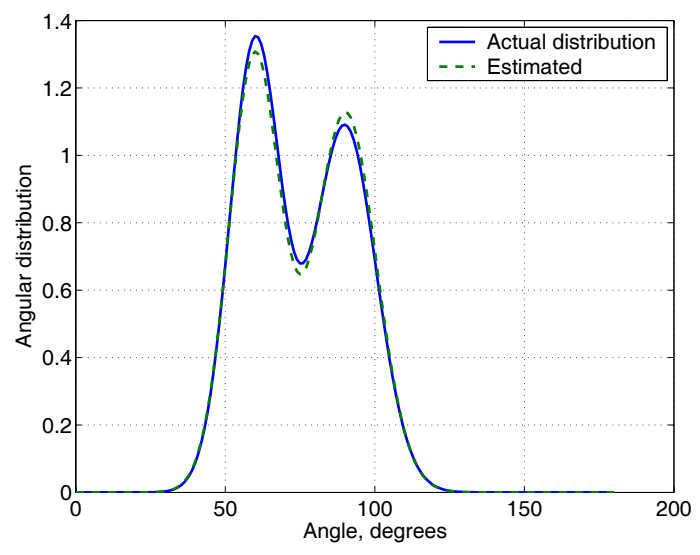

Fig. 2. Comparison of the obtained angular distribution to the actual distribution. Multimodal distribution is modeled with high fidelity.

\section{SIMULATION RESULTS}

In this section we present some simulation results in order to compare the performance of the estimator to the CRLB. In all simulations the receiver has an uniform linear array (ULA) with $N=8$ antennas. We will consider the angle of arrival is the outcome of a mixture of $P$ Von Mises distributions, $P$ known. The received signal is generated as

$$
\mathbf{y}(k)=\mathbf{R}^{1 / 2} \mathbf{w}(k)+\mathbf{n}(k),
$$

where $\mathbf{R}^{1 / 2}$ is obtained by the Cholesky decomposition of the channel covariance matrix, i.e., $E\left[\mathbf{h} \mathbf{h}^{H}\right]=\mathbf{R}^{1 / 2}\left(\mathbf{R}^{1 / 2}\right)^{H}$, and $\mathbf{w}(k)$ is a circular complex white Gaussian process. The signalto-noise ratio (SNR) per antenna is fixed at $20 \mathrm{~dB}$.

The simulation parameters are chosen as $\mu=\{60,90\}$, $\kappa=\{45,30\}$, and $\epsilon=\{0.5,0.5\}$, for $P=2$ clusters. Figure 2 compares the actual angular distribution with the estimate obtained using $N_{s}=1000$ samples.

In Figures 3 and 4 we compare the MSE of the estimates of each parameter to the corresponding CRLB as a function of the number of samples. It can be seen that the MSE reaches the CRLB for small number of samples.

\section{CONCLUSION}

In this paper we proposed a technique presented for estimation of the angular distribution of the diffuse scattering component in channel sounding applications. The method allows estimating the propagation parameters when there are multiple clusters of scatterers present in the environment as in the bad urban scenarios. The method extends the results in $[6,7]$ to these more demanding scenarios. A mixture of angular pdfs model is used and the parameters of each mixture component as well as the mixture proportions are estimated. The large sample properties of the proposed estimator are studied by establishing the CRLB and comparing the variances of the estimates to the bound. The results show that the proposed method possesses desirable optimality properties since the CRLB is attained for the parameters with relatively small sample sizes. 


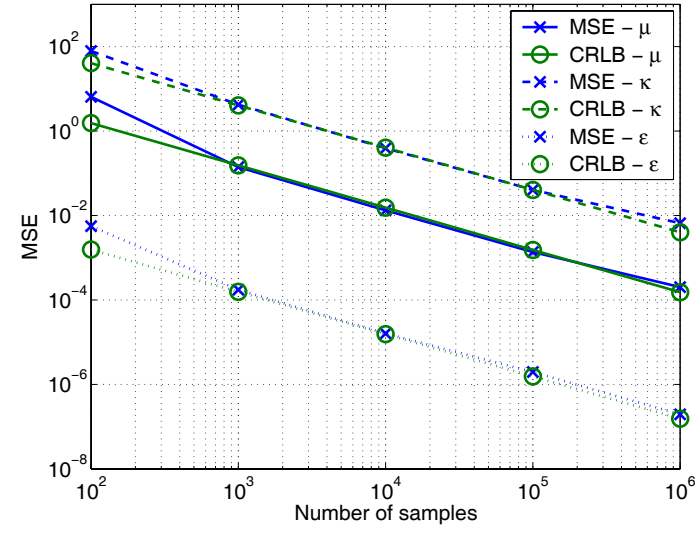

Fig. 3. MSE and CRLB as a function of the number of samples for the cluster with parameters $\mu_{1}=60^{\circ}, \kappa_{1}=45$, and $\epsilon=0.5$. The receive array is an ULA with 8 antennas. SNR is fixed at $20 \mathrm{~dB}$ per antenna. CRLB is reached with relatively small sample sizes.

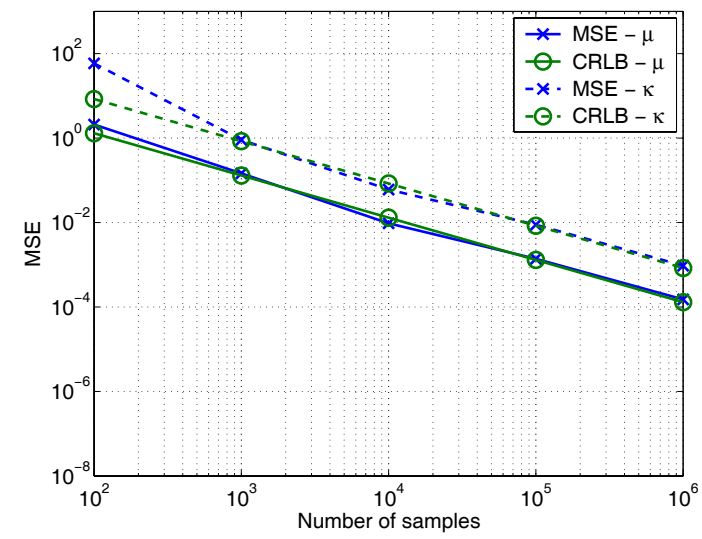

Fig. 4. MSE and CRLB as a function of the number of samples for the cluster with parameters $\mu_{2}=90^{\circ}$ and $\kappa_{2}=30$. The receive array is an ULA with 8 antennas. SNR is fixed at $20 \mathrm{~dB}$ per antenna. CRLB is attained with relatively small sample sizes.
The method is also numerically more stable than commonly used methods based deterministic model with very large number of parameters [1] because of the lower dimensionality and smoother likelihood function.

\section{REFERENCES}

[1] B. H. Fleury, P. Jourdan, and A. Stucki, "High-resolution channel parameter estimation for MIMO applications using the SAGE algorithm," in 2002 International Zurich Seminar on BroadBand Communications. Access, Transmission, Networking, Feb 2002, pp. 30-1 - 30-9.

[2] Q. H. Spencer, B. D. Jeffs, M. A. Jensen, and A. L. Swindlehurst, "Modeling the statistical time and angle of arrival characteristics of an indoor multipath channel," IEEE Journal on Selected Areas in Communications, vol. 18, no. 3, pp. 347360, Mar. 2000.

[3] B. H. Fleury, M. Tschudin, R. Heddergott, D. Dahlhaus, and K. I. Pedersen, "Channel parameter estimation in mobile radio environments using the SAGE algorithm," IEEE Journal on Selected Areas in Communications, vol. 17, no. 3, pp. 434450, March 1999.

[4] A. Abdi and M. Kaveh, "A space-time correlation model for multielement antenna systems in mobile fading channels," IEEE Journal on Selected Areas in Communications, vol. 20, no. 3, pp. 550-560, April 2002.

[5] K. V. Mardia, Statistics of Directional Data, Academic Press, London and New York, 1972.

[6] C. B. Ribeiro, E. Ollila, and V. Koivunen, "Stochastic maximum likelihood method for propagation parameter estimation," in IEEE International Symposium on Personal, Indoor and Mobile Radio Communications, Sep. 2004.

[7] C. B. Ribeiro, E. Ollila, and V. Koivunen, "Cramér-rao bound for angular propagation parameter estimation in MIMO systems," in Asilomar Conference on Signals, Systems, and Computers, Nov. 2004.

[8] K. I. Pedersen, P. E. Mogensen, and B. H. Fleury, "A stochastic model of the temporal and azimuthal dispersion seen at the base station in outdoor propagation environments," IEEE Transactions on Vehicular Technology, vol. 49, no. 2, pp. 437447, March 2000.

[9] R. Fletcher, Practical Methods of Optimization - Constrained Optimization, vol. 2, John Wiley and Sons, Chichester, USA, 1981. 